\title{
Robust cooperative sensor network localization via the EM criterion in LOS/NLOS environments
}

\author{
Feng Yin, Abdelhak M. Zoubir, Carsten Fritsche and Fredrik Gustafsson
}

\section{Linköping University Post Print}

\section{Tweet}

N.B.: When citing this work, cite the original article.

(C)2013 IEEE. Personal use of this material is permitted. However, permission to reprint/republish this material for advertising or promotional purposes or for creating new collective works for resale or redistribution to servers or lists, or to reuse any copyrighted component of this work in other works must be obtained from the IEEE.

Feng Yin, Abdelhak M. Zoubir, Carsten Fritsche and Fredrik Gustafsson, Robust cooperative sensor network localization via the EM criterion in LOS/NLOS environments, 2013, IEEE 14th Workshop on Signal Processing Advances in Wireless Communications (SPAWC), 2013, 505509.

http://dx.doi.org/10.1109/SPAWC.2013.6612101

Postprint available at: Linköping University Electronic Press

http://urn.kb.se/resolve?urn=urn:nbn:se:liu:diva-121627 


\section{Robust Cooperative Sensor Network Localization via the EM Criterion in LOS/NLOS Environments}

\author{
Feng Yin* and Abdelhak M. Zoubir \\ Signal Processing Group \\ Technische Universität Darmstadt \\ Darmstadt, Germany \\ Email: \{fyin, zoubir\}@spg.tu-darmstadt.de
}

\author{
Carsten Fritsche \\ IFEN GmbH \\ Poing, Germany \\ Email: carsten@isy.liu.se
}

\author{
Fredrik Gustafsson \\ Division of Automatic Control \\ Linköping University \\ Linköping, Sweden \\ Email: fredrik@isy.liu.se
}

\begin{abstract}
We investigate robust cooperative localization in LOS/NLOS environments in wireless sensor networks. Roundtrip time-of-arrival signal metric is considered so that time synchronization among sensors can be avoided. Owing to the nonline-of-sight effect, we model the measurement error by a twomode Gaussian mixture distribution. However, its parameters are assumed completely unknown. We propose a centralized localization algorithm, which jointly estimates the unknown geographical coordinates and the nuisance mixture model parameters. The expectation-maximization criterion is adopted here to implement the maximum likelihood estimator. In addition, we also compute the Cramér-Rao lower bound (CRLB) for our estimation problem and present the best achievable positioning accuracy in terms of the CRLB.
\end{abstract}

Keywords-Cramér-Rao lower bound (CRLB), expectationmaximization (EM) criterion, Gaussian mixture, non-line-of-sight (NLOS), robust cooperative localization.

\section{INTRODUCTION}

The use of wireless sensor networks, consisting of tiny, low-cost, low-power, multi-functional sensors, has enabled a wide variety of monitoring and control applications [1]. In many cases, the data collected by a sensor node is worthless unless some knowledge about the sensor's location is provided. In wireless sensor networks, in general, only a small fraction of sensors know their geographical coordinates a priori [2]. In this paper, we refer to this type of sensors as anchors. The rest of sensors, a.k.a. agents, have to determine their own coordinates. In contrast to the conventional infrastructurebased non-cooperative localization (for instance, [3], [4]), the new emerging cooperative localization also exploits inter-agent distance information and generates well improved positioning accuracy as a consequence.

In the last decade, a plethora of cooperative localization algorithms was proposed (see e.g., [5], [6], [7], [8], [9], [10]). An excellent survey of the existing algorithms can be found in [11, Chapter 23]. Motivated by [2], statistical measurement model is a common assumption in a majority of the existing algorithms. In this paper, we consider a specific problem in mixed line-of-sight (LOS)/ non-LOS (NLOS) environments and follow up a statistical round-trip time-of-arrival (RTOA) measurement model so that time-synchronization among sensors can be avoided. In the NLOS environments, the measurement error is reported to have a positive bias and larger variance both in wireless cellular networks [4] and in wireless sensor networks [10]. Measurement errors due to the NLOS propagation (a.k.a. outliers) can deteriorate or paralyze the algorithms developed for LOS environments (assuming Gaussian errors). Towards more robustness, we take the most representative two-mode Gaussian mixture distribution, which has been intensively used for robust non-cooperative localization in wireless cellular networks e.g., [4], [12] and references therein.

In [13], [14], outlier compensation was considered but not in the context of NLOS mitigation. In [13], a robust algorithm was obtained through replacing the least-squares function with Huber's function in the minimization problem originated from [9]. The advantage of this method is that no specific measurement error model needs to be assumed. In [14], a general two-mode mixture measurement error model was assumed and the expectation-maximization (EM) criterion [15] was applied to the estimation problem. In contrast to [14], we assume the mixture model parameters to be completely unknown. This lies in the fact that wireless sensor networks are often deployed in unexplored and inaccessible environments, such as those considered in battlefield surveillance, nuclear/chemical attack detection, and forest fire detection [1], where offline calibration is impossible to be carried out.

Our original contributions of this paper are twofold. First, we jointly estimate the geographical coordinates and the mixture model parameters. A centralized algorithm is developed based on the EM criterion to implement the maximum likelihood estimator (MLE). Secondly, we evaluate the Cramér-Rao lower bound (CRLB) for our estimation problem. This task is more complicated than the one presented in [14], where both the mixture model parameters and the propagation conditions (LOS or NLOS) are assumed known.

The remainder of this paper is organized as follows: In Section II, we introduce the signal model and state the problem at hand. In Section III, we briefly mention the standard maximum likelihood implementation and elaborate on our proposed EM algorithm, followed by the CRLB computation in Section IV. Simulation results are shown in Section V, and conclusions are drawn in Section VI.

\section{Signal Model}

We consider a connected network of $N$ sensors in a twodimensional (2-D) space (although extension to 3-D case is straightforward). It is assumed that the first $N_{u}$ sensors are agents, whose geographical coordinates $\boldsymbol{p}_{i}=\left[x_{i}, y_{i}\right]^{T}$, $i=1,2, \ldots, N_{u}$ are unknown; and the remaining $N-N_{u}$ sensors are anchors with perfect a priori knowledge about their positions $\boldsymbol{p}_{i}, i=N_{u}+1, N_{u}+2, \ldots, N$. 
In this paper, we assume that the neighboring sensors make pair-wise RTOA measurements. Here, we define $\mathcal{H}(i)$ to indicate the set of neighboring sensors with which the $i$ th agent, $i=1,2, \ldots, N_{u}$ conducts RTOA measurements. Moreover, we make the following assumptions: (1) $i \notin \mathcal{H}(i)$; (2) $j \in \mathcal{H}(i)$ if and only if $i \in \mathcal{H}(j)$; (3) the $i$ th agent measures RTOA, $t_{i, j}$, with every sensor whose index $j$ satisfies $j \in \mathcal{H}(i)$ and $j>i$. The above assumptions are similar to those made in [6].

In the sequel, we correspond RTOA measurements to range measurements. The relation between RTOA and range metrics can be found in [10]. The range measurement $r_{i, j}$ made between any pair of neighboring sensors $(i, j)$ is given by

$$
r_{i, j}=d\left(\boldsymbol{p}_{i}, \boldsymbol{p}_{j}\right)+v_{i, j}
$$

where

$$
d\left(\boldsymbol{p}_{i}, \boldsymbol{p}_{j}\right)=\sqrt{\left(x_{i}-x_{j}\right)^{2}+\left(y_{i}-y_{j}\right)^{2}}
$$

is the true Euclidean distance between the two sensors and $v_{i, j}$ is the measurement error. Here, we assume the measurement errors observed for different sensor pairs are independent and identically distributed (i.i.d.) as

$$
p_{V}(v)=\alpha_{1} \mathcal{N}\left(v ; \mu_{1}, \sigma_{1}^{2}\right)+\alpha_{2} \mathcal{N}\left(v ; \mu_{2}, \sigma_{2}^{2}\right)
$$

meaning that a measurement error $v$ is distributed according to $\mathcal{N}\left(v ; \boldsymbol{\beta}_{1}\right)$ with probability $\alpha_{1}$ in LOS environments or to $\mathcal{N}\left(v ; \boldsymbol{\beta}_{2}\right)$ with probability $\alpha_{2}$ in NLOS environments [4]. In the literature, $\alpha_{2}$ is also known as the "NLOS contamination ratio". Here, we assume the mixture model parameters $\alpha_{l}$, $\boldsymbol{\beta}_{l}=\left[\mu_{l}, \sigma_{l}^{2}\right]^{T}, l=1,2$ are completely unknown.

The objective of this paper is to jointly estimate the geographical coordinates $\boldsymbol{p}_{i}=\left[x_{i}, y_{i}\right]^{T}, i=1,2, \ldots, N_{u}$ of the agents as well as the mixture model parameters $\alpha_{l}, \boldsymbol{\beta}_{l}$, $l=1,2$ based on the pair-wise range measurements $r_{i, j}$ made between all neighboring sensors and the known geographical coordinates of the anchors. In the sequel, we let $\boldsymbol{\theta}=\left[\alpha_{1}, \alpha_{2}, \boldsymbol{\beta}_{1}^{T}, \boldsymbol{\beta}_{2}^{T}, \boldsymbol{p}_{x y}^{T}\right]^{T}$ be a vector of unknown parameters in which $\boldsymbol{p}_{x y}=\left[x_{1}, x_{2}, \ldots, x_{N_{u}}, y_{1}, y_{2}, \ldots, y_{N_{u}}\right]^{T}$ includes the geographical coordinates of the agents and let $\mathcal{S}$ be a set of all feasible sensor pairs $(i, j)$ for which range measurements $r_{i, j}$ are obtained and stacked in a vector $r$. The dimension of $\boldsymbol{r}$ is equivalent to the cardinality of the set $\mathcal{S}$, namely $|\mathcal{S}|$.

\section{MAXIMUM LIKELIHOOD ESTIMATION}

The maximum-likelihood estimator of $\boldsymbol{\theta}$ globally maximizes the log-likelihood function, and it is favorable for its asymptotic properties [16, Theorem 7.3]. In this paper, we aim at finding the MLE of $\boldsymbol{\theta}$. To that end, we express the log-likelihood function of our estimation problem as follows:

$$
\begin{aligned}
\mathcal{L}_{i}(\boldsymbol{\theta} ; \boldsymbol{r}) & =\ln p(\boldsymbol{r} ; \boldsymbol{\theta})=\sum_{i=1}^{N_{u}} \sum_{\substack{j>i \\
j \in \mathcal{H}(i)}}^{N} \ln p\left(r_{i, j} ; \boldsymbol{\theta}\right), \\
& =\sum_{(i, j) \in \mathcal{S}} \ln \left(\sum_{l=1}^{2} \alpha_{l} \mathcal{N}\left(r_{i, j}-d_{i, j} ; \boldsymbol{\beta}_{l}\right)\right) .
\end{aligned}
$$

Note that in (4), $d_{i, j}$ is a short-hand notation of $d\left(\boldsymbol{p}_{i}, \boldsymbol{p}_{j}\right)$. The conventional way of generating the MLE is through solving:

$$
\begin{array}{ll}
\underset{\boldsymbol{\theta}}{\operatorname{maximize}} & \mathcal{L}_{i}(\boldsymbol{\theta} ; \boldsymbol{r}) \\
\text { subject to } & 0<\alpha_{1}, \alpha_{2}<1, \alpha_{1}+\alpha_{2}=1, \\
& \sigma_{1}^{2}>0, \sigma_{2}^{2}>0 .
\end{array}
$$

This optimization problem is cumbersome due to "the logarithm of the sum" in the objective function.

Instead of solving (5) directly, we take a detour to approximate the MLE using the EM criterion. That is, we introduce a complete data set $\{\boldsymbol{z}, \boldsymbol{r}\}$ in which $\boldsymbol{z}$ is a column vector enclosing a number of $|\mathcal{S}|$ latent variables $z_{i, j}$ whose values reveal which mixture component is responsible for the corresponding range measurement $r_{i, j},(i, j) \in \mathcal{S}$. The latent variables inherit the independent property from the range measurements. As a result, the complete data log-likelihood function is given by

$$
\mathcal{L}_{c}(\boldsymbol{\theta} ; \boldsymbol{z}, \boldsymbol{r})=\sum_{(i, j) \in \mathcal{S}} \ln \left(\alpha_{z_{i, j}} \mathcal{N}\left(r_{i, j}-d_{i, j} ; \boldsymbol{\beta}_{z_{i, j}}\right)\right),
$$

to which the EM criterion will be ultimately applied. The resulting iterative algorithm is called EM algorithm in the remaining parts of this paper. Given the a priori parameter estimate $\boldsymbol{\theta}^{(\eta)}$, we show the workflow of the proposed EM algorithm on the $(\eta+1)$ th iteration below.

Expectation Step: We perform expectation of the complete data log-likelihood with respect to $z$, namely,

$$
Q\left(\boldsymbol{\theta} ; \boldsymbol{\theta}^{(\eta)}\right)=\sum_{\boldsymbol{z} \in \boldsymbol{\Upsilon}} \mathcal{L}_{c}(\boldsymbol{\theta} ; \boldsymbol{z}, \boldsymbol{r}) \operatorname{Pr}\left\{\boldsymbol{z} \mid \boldsymbol{r} ; \boldsymbol{\theta}^{(\eta)}\right\}
$$

where $\boldsymbol{\Upsilon}$ is the parameter space of $\boldsymbol{z}$ and $\operatorname{Pr}\left\{\boldsymbol{z} \mid \boldsymbol{r} ; \boldsymbol{\theta}^{(\eta)}\right\}$ is the conditional probability of the latent variables $z$. Following the same procedure for simplifying $Q\left(\boldsymbol{\theta} ; \boldsymbol{\theta}^{(\eta)}\right)$ in [17], we obtain

$$
Q\left(\boldsymbol{\theta} ; \boldsymbol{\theta}^{(\eta)}\right)=\sum_{(i, j) \in \mathcal{S}} \sum_{l=1}^{2} \ln \left(\alpha_{l} \mathcal{N}\left(r_{i, j}-d_{i, j} ; \boldsymbol{\beta}_{l}\right)\right) \tilde{P}_{l, i, j}^{(\eta)}
$$

where $\tilde{P}_{l, i, j}^{(\eta)}$ is a short-hand notation of the conditional probability $\operatorname{Pr}\left\{z_{i, j}=l \mid r_{i, j} ; \boldsymbol{\theta}^{(\eta)}\right\}$. With the help of Bayes' rule, we can easily calculate

$$
\tilde{P}_{l, i, j}^{(\eta)}=\frac{\alpha_{l}^{(\eta)} \mathcal{N}\left(r_{i, j}-d_{i, j}^{(\eta)} ; \boldsymbol{\beta}_{l}^{(\eta)}\right)}{p\left(r_{i, j} ; \boldsymbol{\theta}^{(\eta)}\right)} .
$$

Maximization Step: We attempt to maximize $Q\left(\boldsymbol{\theta} ; \boldsymbol{\theta}^{(\eta)}\right)$, c.f. (8), with respect to $\boldsymbol{\theta}=\left[\alpha_{1}, \alpha_{2}, \boldsymbol{\beta}_{1}^{T}, \boldsymbol{\beta}_{2}^{T}, \boldsymbol{p}_{x y}^{T}\right]^{T}$. For better demonstration of the maximization procedure, we re-express $Q\left(\boldsymbol{\theta} ; \boldsymbol{\theta}^{(\eta)}\right)$ by

$$
Q\left(\boldsymbol{\theta} ; \boldsymbol{\theta}^{(\eta)}\right)=Q_{0}^{(\eta)}\left(\alpha_{1}, \alpha_{2}\right)+\sum_{l=1}^{2} Q_{l}^{(\eta)}\left(\boldsymbol{\beta}_{l}, \boldsymbol{p}_{x y}\right)
$$

where

$$
Q_{0}^{(\eta)}\left(\alpha_{1}, \alpha_{2}\right)=\sum_{(i, j) \in \mathcal{S}} \ln \left(\alpha_{1}\right) \tilde{P}_{1, i, j}^{(\eta)}+\ln \left(\alpha_{2}\right) \tilde{P}_{2, i, j}^{(\eta)},
$$

and for $l=1,2$,

$$
Q_{l}^{(\eta)}\left(\boldsymbol{\beta}_{l}, \boldsymbol{p}_{x y}\right)=\sum_{(i, j) \in \mathcal{S}} \ln \left(\mathcal{N}\left(r_{i, j}-d_{i, j} ; \boldsymbol{\beta}_{l}\right)\right) \tilde{P}_{l, i, j}^{(\eta)} .
$$


We first update the mixture model parameters through solving the following equations:

$$
\begin{gathered}
\frac{\partial}{\partial \alpha_{l}}\left[Q_{0}^{(\eta)}\left(\alpha_{1}, \alpha_{2}\right)+\lambda\left(\sum_{l=1}^{2} \alpha_{l}-1\right)\right]=0, \\
\frac{\partial}{\partial \mu_{l}}\left[Q_{l}^{(\eta)}\left(\mu_{l}, \sigma_{l}^{2}, \boldsymbol{p}_{x y}^{(\eta)}\right)\right]=0 \\
\frac{\partial}{\partial \sigma_{l}^{2}}\left[Q_{l}^{(\eta)}\left(\mu_{l}^{(\eta+1)}, \sigma_{l}^{2}, \boldsymbol{p}_{x y}^{(\eta)}\right)\right]=0
\end{gathered}
$$

and the corresponding solutions are given in analytical form as follows:

$$
\begin{gathered}
\alpha_{l}^{(\eta+1)}=\frac{1}{|\mathcal{S}|} \sum_{(i, j) \in \mathcal{S}} \tilde{P}_{l, i, j}^{(\eta)} \\
\mu_{l}^{(\eta+1)}=\frac{\sum_{(i, j) \in \mathcal{S}}\left(r_{i, j}-d\left(\boldsymbol{p}_{i}^{(\eta)}, \boldsymbol{p}_{j}^{(\eta)}\right)\right) \tilde{P}_{l, i, j}^{(\eta)}}{|\mathcal{S}| \cdot \alpha_{l}^{(\eta+1)}} \\
\sigma_{l}^{2,(\eta+1)}=\frac{\sum_{(i, j) \in \mathcal{S}}\left(r_{i, j}-d\left(\boldsymbol{p}_{i}^{(\eta)}, \boldsymbol{p}_{j}^{(\eta)}\right)-\mu_{l}^{(\eta+1)}\right)^{2} \tilde{P}_{l, i, j}^{(\eta)}}{|\mathcal{S}| \cdot \alpha_{l}^{(\eta+1)}} .
\end{gathered}
$$
by

Next, geographical coordinates of the agents are updated

$$
\boldsymbol{p}_{x y}^{(\eta+1)}=\arg \max _{\boldsymbol{p}_{x y}} \sum_{l=1}^{2} Q_{l}^{(\eta)}\left(\boldsymbol{\beta}_{l}^{(\eta+1)}, \boldsymbol{p}_{x y}\right),
$$

which boils down to minimizing

$$
f\left(\boldsymbol{p}_{x y}\right)=\sum_{(i, j) \in \mathcal{S}} \sum_{l=1}^{2} \frac{\left(r_{i, j}-d\left(\boldsymbol{p}_{i}, \boldsymbol{p}_{j}\right)-\mu_{l}^{(\eta+1)}\right)^{2}}{2 \sigma_{l}^{2,(\eta+1)}} \tilde{P}_{l, i, j}^{(\eta)}
$$

where $\mu_{1}^{(\eta+1)}, \mu_{2}^{(\eta+1)}, \sigma_{1}^{2,(\eta+1)}, \sigma_{2}^{2,(\eta+1)}$ are substitutions of the corresponding unknown parameters, respectively. Since $d\left(\boldsymbol{p}_{i}, \boldsymbol{p}_{j}\right)$ is non-linear in terms of both $x_{i(j)}$ and $y_{i(j)}$, we resolve (20) numerically via a BFGS quasi-Newton method as described in [18]. It can be shown that the proposed EM algorithm converges monotonically to some stationary point $\mathcal{L}^{*}$ of the log-likelihood function $\mathcal{L}_{i}(\boldsymbol{\theta} ; \boldsymbol{r})$. Here, a stationary point could be a saddle point, a local maximum or a global maximum. The proof of the convergence will be given in great detail in a forthcoming paper. As a conclusion of this section, we summarize the key steps of the proposed EM algorithm in Algorithm 1.

\section{CRAMÉr-RAO LOWER BOUnd COMPUTATION}

In the case of Gaussian measurement error, the CramérRao lower bound (CRLB) exists in closed form for various network position estimation problems, for instance, [6], [19]. However, CRLB computation for analogous problems in nonGaussian and impulsive measurement error is rarely considered in the literature. In order to compute the CRLB for our original estimation problem in (5), we resort to a numerical method.

We start by expressing the Fisher information matrix (FIM) as

$$
\mathcal{F}(\boldsymbol{\theta})=\mathbb{E}_{p(\boldsymbol{r} ; \boldsymbol{\theta})}\left\{-\Delta_{\boldsymbol{\theta}}^{\boldsymbol{\theta}} \ln p(\boldsymbol{r} ; \boldsymbol{\theta})\right\}
$$

Algorithm 1 EM Algorithm for Joint Parameter Estimation Initialization: Define the convergence tolerance $\Delta$; Choose an initial guess $\boldsymbol{\theta}^{(\eta=0)}=\left[\alpha_{1}^{(0)}, \alpha_{2}^{(0)}, \mu_{1}^{(0)}, \mu_{2}^{(0)}, \sigma_{1}^{2,(0)}, \sigma_{2}^{2,(0)}, \boldsymbol{p}_{x y}^{T,(0)}\right]^{T}$.

Expectation and Maximization:

In the $(\eta+1)$ th iteration $(\eta \in \mathbb{Z}, \eta \geq 0)$, do:

1) Compute $\tilde{P}_{l, i, j}^{(\eta)}, \forall(i, j) \in \mathcal{S}$ and $l=1,2$, according to (9);

2) Evaluate $\alpha_{l}^{(\eta+1)}, \mu_{l}^{(\eta+1)}, \sigma_{l}^{2,(\eta+1)}, l=1,2$ according to (16), (17), and (18), respectively;

3) Find $\boldsymbol{p}_{x y}^{(\eta+1)}$ through minimizing $f\left(\boldsymbol{p}_{x y}\right)$, cf. (20), via the BFGS quasi-Newton method initialized by $\boldsymbol{p}_{x y}^{(\eta)}$.

\section{Convergence Check:}

If $\left|\mathcal{L}_{i}\left(\boldsymbol{\theta}^{(\eta+1)} ; \boldsymbol{r}\right)-\mathcal{L}_{i}\left(\boldsymbol{\theta}^{(\eta)} ; \boldsymbol{r}\right)\right| \leq \Delta$, then stop; otherwise reset $\eta \leftarrow$ $\eta+1$ and return to the EM stage.

where the expectation is taken with respect to $p(\boldsymbol{r} ; \boldsymbol{\theta})$. In our problem, it is more convenient to express the FIM as follows:

$$
\begin{aligned}
\mathcal{F}(\boldsymbol{\theta}) & =\mathbb{E}_{p(\boldsymbol{r} ; \boldsymbol{\theta})}\left\{\nabla_{\boldsymbol{\theta}} \ln p(\boldsymbol{r} ; \boldsymbol{\theta}) \cdot \nabla_{\boldsymbol{\theta}}^{T} \ln p(\boldsymbol{r} ; \boldsymbol{\theta})\right\} \\
& =\int\left\{\nabla_{\boldsymbol{\theta}} \ln p(\boldsymbol{r} ; \boldsymbol{\theta}) \cdot \nabla_{\boldsymbol{\theta}}^{T} \ln p(\boldsymbol{r} ; \boldsymbol{\theta})\right\} p(\mathbf{r} ; \boldsymbol{\theta}) \mathrm{dr} .
\end{aligned}
$$

Due to the difficulty in evaluating the integration in (22) in an analytical manner, we resort to Monte Carlo integration techniques [20], yielding

$$
\mathcal{F}(\boldsymbol{\theta}) \approx \frac{1}{N_{M}} \sum_{n=1}^{N_{M}}\left\{\nabla_{\boldsymbol{\theta}} \ln p\left(\boldsymbol{r}^{(n)} ; \boldsymbol{\theta}\right) \cdot \nabla_{\boldsymbol{\theta}}^{T} \ln p\left(\boldsymbol{r}^{(n)} ; \boldsymbol{\theta}\right)\right\}
$$

where $\boldsymbol{r}^{(n)}, n=1,2, \ldots, N_{M}$ are generated as follows. First, we generate a vector $\boldsymbol{v}^{(n)}$ comprising i.i.d. measurement errors $v_{i, j}^{(n)}, \forall(i, j) \in \mathcal{S}$ from the given two-mode Gaussian mixture distribution. Next, a vector $\boldsymbol{r}^{(n)}$ can be easily obtained with the help of (1). The computation of $\nabla_{\boldsymbol{\theta}} \ln p(\boldsymbol{r} ; \boldsymbol{\theta})$ is straightforward and thus is omitted here due to space limitations. The CRLB is obtained as the inverse of the FIM, namely,

$$
\operatorname{CRLB}(\boldsymbol{\theta})=\mathcal{F}^{-1}(\boldsymbol{\theta}) .
$$

In [14], the CRLB is calculated for the case that both the mixture model parameters and the propagation conditions (i.e., $\left.z_{i, j}, \forall(i, j) \in \mathcal{S}\right)$ are assumed perfectly known. However, the latter assumption is unrealistic. In fact, even without the known propagation conditions, a more convincing CRLB can still be obtained by following a similar procedure as mentioned in [12]. The idea is, still, to numerically evaluate the FIM using Monte-Carlo integration techniques. But in this case, the computational cost can be extensively reduced as compared to that consumed in evaluating (23).

We evaluate the positioning accuracy in terms of the overall root mean square error (RMSE), which is defined by

$$
\overline{\mathrm{RMSE}}=\frac{1}{N_{u}} \sum_{i=1}^{N_{u}} \sqrt{E\left[\left(\hat{x}_{i}-x_{i}\right)^{2}+\left(\hat{y}_{i}-y_{i}\right)^{2}\right]}
$$

where $\left[\hat{x}_{i}, \hat{y}_{i}\right]$ is any unbiased estimator of the $i$ th agent's true 2-D geographical coordinates $\left[x_{i}, y_{i}\right]$. Clearly, the fundamental limits of $\overline{\mathrm{RMSE}}$ is given by

$$
\overline{\operatorname{RMSE}} \geq \mathrm{CRLB}_{\mathrm{pos}}=\frac{1}{N_{u}} \sum_{i=1}^{N_{u}} \sqrt{\operatorname{CRLB}\left(x_{i}\right)+\operatorname{CRLB}\left(y_{i}\right)} .
$$

In other words, $\mathrm{CRLB}_{\text {pos }}$ places a lower bound on $\overline{\mathrm{RMSE}}$. 


\section{Simulation Results}

We simulate a network of $N=50$ sensors, among which $N_{u}=41$ sensors are agents and the others are anchors. The geographical coordinates of the agents are uniformly deployed in a 60 -meter by 60 -meter area in a 2 -D space. Throughout this section, we consider a particular sensor network depicted in Fig. 1. Following [2], we determine the set of neighboring sensors $H(i)$ according to the radius of connectivity $R_{c}$. This is merely for ease of the simulation, since the sensors don't really know which sensors are inside $R_{c}$. A realistic way of doing this in the practice is to compare the received signal strength with a predetermined threshold. Here, we assume $\boldsymbol{\beta}_{1}=\left[\mu_{1}=0, \sigma_{1}^{2}=4\right]^{T}$ and $\boldsymbol{\beta}_{\mathbf{2}}=\left[\mu_{2}=15, \sigma_{2}^{2}=9\right]^{T}$. In the sequel, we somewhat simplify our estimation problem by assuming that $\mu_{1}$ is already known, since many experimental campaigns have confirmed this in different LOS environments.

In addition to the newly proposed EM algorithm, we also demonstrate the positioning performance of a least-squares (LS) algorithm and a robust-M estimation algorithm. The LS algorithm estimates the unknown geographical coordinates through solving

$$
\arg \min _{\boldsymbol{p}_{x y}} \sum_{(i, j) \in \mathcal{S}}\left(r_{i, j}-d\left(\boldsymbol{p}_{i}, \boldsymbol{p}_{j}\right)\right)^{2} .
$$

For enhanced robustness against the outliers, the LS function in (27) is replaced with Huber's score function in the robust$\mathrm{M}$ estimation algorithm. These two algorithms are simplified versions of those proposed in [9] and [13], respectively, where an additional penalty term concerning some a priori knowledge about the agent's position was added in the minimization problems.

Throughout the simulations, our proposed EM algorithm assumes $\alpha_{1}^{(0)}=\alpha_{2}^{(0)}=0.5, \mu_{2}^{(0)}=10, \sigma_{1}^{2,(0)}=\sigma_{2}^{2,(0)}=8$ and $\boldsymbol{p}_{x y}^{(0)}$ is equal to the true parameter contaminated by $\boldsymbol{\Delta}_{x y}$ whose elements are generated from a uniform distribution $\mathcal{U}(0,15)$ (in meter). In practical applications, an initial guess can be determined adaptively in a two step procedure. In the first step, $\boldsymbol{p}_{x y}^{(0)}$ is obtained as an output of the conventional multidimensional scaling (MDS) algorithm [7]; and in the second step, we initialize the mixture model parameters using the results derived in [21, Sec. 1.4.16]. In order to create a distance matrix in the MDS algorithm, we have to replace the range measurement with the minimum multi-hop distance if two sensors are not neighbors and assume $r_{i, j}=r_{j, i}$. Moreover, we set the convergence tolerance $\Delta=0.01$.

In the first example of our simulation, we illustrate how the NLOS contamination ratio will influence the positioning accuracy of different algorithms. To this end, we calculate $\overline{\mathrm{RMSE}}$ as a function of $\alpha_{2}$, respectively for the LS algorithm, the robust-M algorithm, and the EM algorithm. Here, we let $\alpha_{2}$ vary from 0.1 to 0.9 and let $R_{c}=30$ meter, resulting $|\mathcal{S}|=503$ neighboring pairs for the selected sensor network. The $\overline{\text { RMSE }}$ in (25) is approximated in a Monte-Carlo experiment with 500 independent trials. Moreover, the best achievable positioning accuracy CRLB $_{\text {pos }}$ is also evaluated according to (26). The results are drawn in Fig. 2. It is obvious that the newly proposed EM algorithm shows the best RMSE curve, however, it doesn't perfectly coincide with the $\mathrm{CRLB}_{\text {pos }}$ curve. The reason is that the EM algorithm only achieves a local maximum or a saddle point in some Monte-Carlo trails, but luckily, the

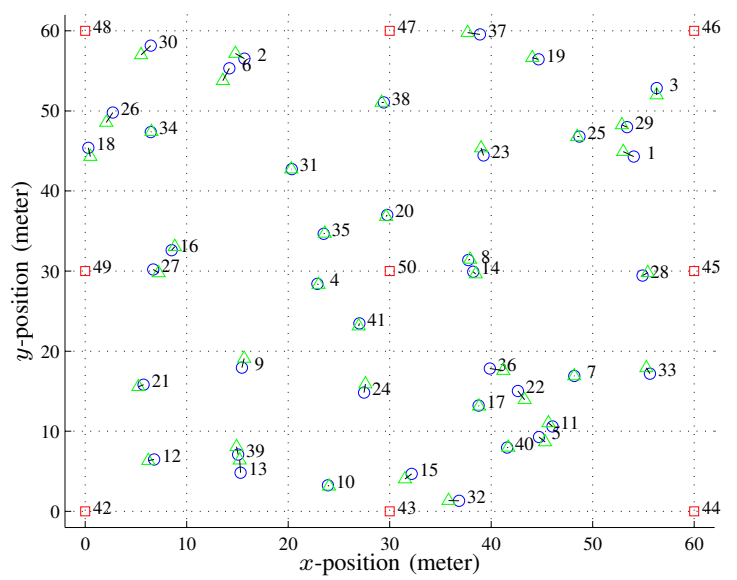

Fig. 1. A sample wireless sensor network as well as a realization of the estimated positions of the agents. Herein, $\square$ 's denote the anchors; o's denote the agents with true coordinates; $\triangle$ 's denote the agents with the estimated coordinates generated by the proposed EM algorithm in a particular MonteCarlo trial with $\alpha_{2}=0.2$ and $R_{c}=30$ meter; and -'s represent the localization errors between the estimated positions and the true positions.

amount of such trials is quite small. The robust-M estimation algorithm is superior to the LS algorithm, since the effect of outliers is somewhat mitigated. But they both generate largely deteriorated positioning accuracy as the NLOS contamination ratio increases. For better demonstration, we also depict in Fig. 1 an estimate of the unknown geographical coordinates obtained for the proposed EM algorithm in one particular Monte-Carlo trail with $\alpha_{2}=0.2$. Clearly, the agents are all accurately localized.

In the second example, we calculate $\overline{\mathrm{RMSE}}$ as a function of the radius of connectivity, $R_{c}$, for the three algorithms. Here, we fix $\alpha_{2}=0.2$. Similarly, we numerically evaluate the $\overline{\mathrm{RMSE}}$ in a Monte-Carlo experiment. The results are shown along with the $\mathrm{CRLB}_{\text {pos }}$ in Fig. 3. We observed that the values of both $\overline{\mathrm{RMSE}}$ and $\mathrm{CRLB}_{\text {pos }}$ monotonically decrease until $R_{c}$ grows to a point beyond which all sensor pairs make measurements. This can be explained from a Fisher information theoretic point of view. As $R_{c}$ increases, more sensor pairs conduct measurements, leading to more information about the relative positions. Besides, we also observed that the risk of getting stuck in a local maximum or a saddle point is higher for the EM algorithm when we have insufficient number of measurements, e.g. $|\mathcal{S}|=254$ for the case that $R_{c}=20$. Nevertheless, the positioning performance does not deviate too much from the lower bound and is still superior to that of the others.

Although not shown in the paper, our simulation results also corroborate that the EM estimator is approximately unbiased. Together with the results shown in the last two examples, we conclude that in most cases the proposed EM algorithm is able to reproduce the MLE.

\section{CONCLUSION}

We proposed a robust EM based cooperative localization algorithm for enhanced positioning accuracy in mixed LOS/NLOS environments. Given a good initial guess, the proposed EM algorithm is able to reproduce the MLE of the unknown parameters, namely the geographical coordi- 


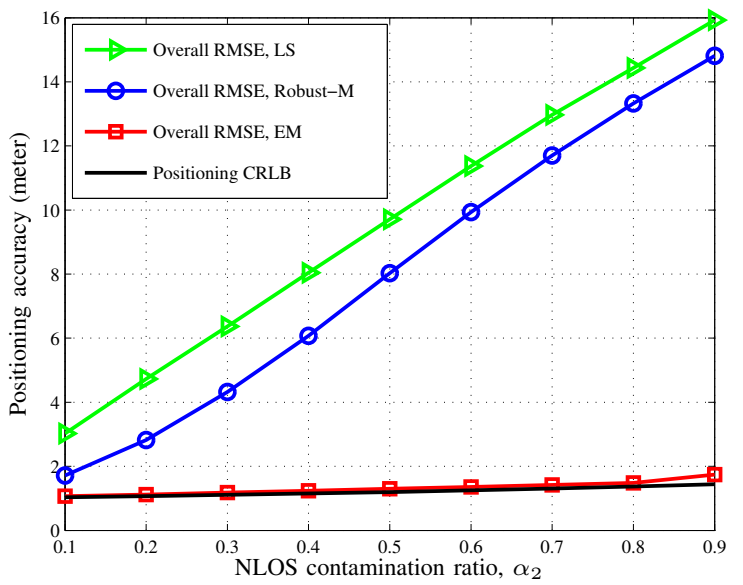

Fig. 2. The overall RMSE of three different sensor network localization algorithms versus the NLOS contamination ratio. Here, the radius of connectivity is fixed to 30 meter.

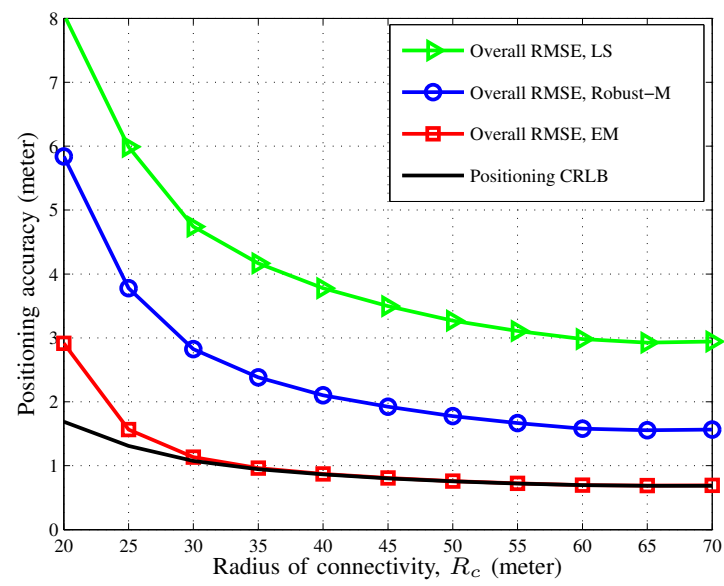

Fig. 3. The overall RMSE of three different sensor network localization algorithms versus the radius of connectivity. Here, the NLOS contamination ratio is fixed to 0.2 .

nates of the agents and the mixture model parameters. A centralized algorithm is considered, since it generally provides more accurate results. Despite the scalability problem, its performance serves as a benchmark for evaluating more attractive distributed implementations for the same estimation problem. Simulation results demonstrate that the positioning accuracy of the proposed EM algorithm largely outperforms two competitors and approaches the fundamental limits. The drawback of the EM algorithm is that the global maximum (or the desired MLE) is not always reachable.

\section{REFERENCES}

[1] I. F. Akyildiz, W. Su, Y. Sankarasubramaniam, and E. Cayirci, "Wireless sensor networks: a survey," Comput. Networks J., vol. 38, pp. 393-422, Mar. 2002.

[2] N. Patwari, J. N. Ash, S. Kyperountas, A. O. Hero III, R. L. Moses, and N. S. Correal, "Locating the nodes: cooperative localization in wireless sensor networks," IEEE Signal Processing Mag., vol. 22, no. 4, pp. 54-69, Jul. 2005.
[3] A. H. Sayed, A. Tarighat, and N. Khajehnouri, "Network-based wireless location: challenges faced in developing techniques for accurate wireless location information," IEEE Signal Processing Mag., vol. 22, no. 4, pp. 24-40, Jul. 2005.

[4] F. Gustafsson and F. Gunnarsson, "Mobile positioning using wireless networks: possibilities and fundamental limitations based on available wireless network measurements," IEEE Signal Processing Mag., vol. 22, no. 4, pp. 41-53, Jul. 2005.

[5] A. Savvides, C.-C. Han, and M. B. Strivastava, "Dynamic fine-grained localization in ad-hoc networks of sensors," in Proc. IEEE Mobicom, 2001, pp. 166-179.

[6] N. Patwari, A. O. Hero III, M. Perkins, N. S. Correal, and R. J. O'Dea, "Relative location estimation in wireless sensor networks," IEEE Trans. Signal Process., vol. 51, no. 8, pp. 2137-2148, Aug. 2003.

[7] Y. Shang, W. Rumi, Y. Zhang, and M. Fromherz, "Localization from connectivity in sensor networks," IEEE Trans. Parallel Distrib. Syst., vol. 15, no. 11, pp. 961-974, Nov. 2004.

[8] A. T. Ihler, J. W. Fisher III, R. L. Moses, and A. S. Willsky, "Nonparametric belief propagation for self-localization of sensor networks," IEEE J. Sel. Areas Commun., vol. 23, no. 4, pp. 809-819, Apr. 2005.

[9] J. A. Costa, N. Patwari, and A. O. Hero III, "Distributed weightedmultidimensional scaling for node localization in sensor networks," ACM Trans. Sen. Netw., vol. 2, no. 1, pp. 39-64, Feb. 2006.

[10] H. Wymeersch, J. Lien, and M. Z. Win, "Cooperative localization in wireless networks," Proc. IEEE, vol. 97, no. 2, pp. 427-450, Feb. 2009.

[11] R. Zekavat and R. M. Buehrer, Handbook of Position Location. Hoboken, NJ: John Wiley \& Sons, Inc., 2011.

[12] F. Yin, C. Fritsche, F. Gustafsson, and A. M. Zoubir, "TOA based robust wireless geolocation and Cramér-Rao lower bound analysis in harsh LOS/NLOS environments," IEEE Trans. Signal Process., accepted for publication, 2013.

[13] S. Korkmaz and A. J. van der Veen, "Robust localization in sensor networks with iterative majorization techniques," in Proc. IEEE Int. Conf. Acoustics, Speech and Signal Processing (ICASSP), Apr. 2009, pp. 2049-2052.

[14] J. N. Ash and R. L. Moses, "Outlier compensation in sensor network self-localization via the EM algorithm," in Proc. IEEE Int. Conf. Acoustics, Speech and Signal Processing (ICASSP), vol. 4, Mar. 2005, pp. 749-752.

[15] A. P. Dempster, N. Laird, and D. B. Rubin, "Maximum likelihood from incomplete data via the EM algorithm," J. Roy. Statist. Soc. B, vol. 39, pp. 1-38, 1977.

[16] S. M. Kay, Fundamentals of Statistical Signal Processing: Estimation Theory. Englewood Cliffs, NJ: Prentice-Hall, Inc., 1993.

[17] J. A. Bilmes, "A gentle tutorial on the EM algorithm and its application to parameter estimation for Gaussian mixture and hidden Markov models," University of California, Berkeley, Berkeley, CA, USA, Tech. Rep., 1998.

[18] F. Yin and A. M. Zoubir, "Robust positioning in NLOS environments using nonparametric adaptive kernel density estimation," in Proc. IEEE Int. Conf. Acoustics, Speech and Signal Processing (ICASSP), Mar. 2012, pp. 3517-3520.

[19] E. G. Larsson, "Cramér-Rao bound analysis of distributed positioning in sensor networks," IEEE Signal Process. Lett., vol. 11, no. 3, pp. 334-337, Mar. 2004.

[20] C. P. Robert and G. Casella, Monte Carlo Statistical Methods. New York, NY: Springer-Verlag, 1999.

[21] Y. Bar-Shalom, X. R. Li, and T. Kirubarajan, Estimation with Applications to Tracking and Navigation. New York, NY: John Wiley \& Sons, Inc., 2001. 